ECLETICA A

Volume 28 (2003) número 1

UNESP

$\underline{\text { www.scielo.br/eq }}$

\title{
GENERALIZAÇÃO DE UM MODELO LINEAR DE IMPEDÂNCIA ELETROQUÍMICA
}

\author{
J. M. V. CAPELA' ${ }^{1}$ M. V. CAPELA ${ }^{1}$; R. MAGNANI
}

\section{Resumo}

Em geral, a função de um modelo de impedância para processos de eletrodo simples é deduzida de um modelo elétrico equivalente, denominado circuito de Randles. Neste trabalho estudou-se a generalização dessa função, mediante a introdução de um parâmetro não-elétrico, relacionado com a flexibilidade do ângulo de fase e da magnitude. A função foi ajustada às medidas experimentais de impedância obtidas de um sistema constituído de uma liga $\mathrm{T}_{\mathrm{i}}-10 \% \mathrm{Al}(\mathrm{m} / \mathrm{m})$ em solução de cloreto de sódio $0,9 \%$, variando-se a amplitude de perturbação. Verificou-se que a função generalizada foi adequada para descrever a impedância do sistema analisado, reduzindo as distorções entre a curva experimental e a curva teórica. Além disso, os melhores resultados foram obtidos para sinais de perturbação do sistema com amplitude igual a $10 \mathrm{mV}$.

Palavras-chave: Impedância eletroquímica; sistemas lineares; regressão multiresposta; ligas de Ti.

\section{Introdução}

A espectroscopia de impedância eletroquímica é uma técnica utilizada na análise de processos eletroquímicos que ocorrem na interface eletrodo/solução eletrolítica. Trata-se de um método de identificação e determinação de parâmetros de um modelo elaborado com base na resposta de freqüência do sistema eletroquímico em estudo.

Em tais experimentos é utilizado um Analisador de Resposta de Freqüência, acoplado a uma interface eletroquímica, o qual mede a resposta na corrente do sistema, à medida que se altera a freqüência de um sinal senoidal de entrada [1,7,11]:

$E(t)=E \operatorname{sen}(w t)$,
Se a amplitude do sinal (1) for suficientemente pequena (da ordem de milivolts), então é possível supor que a resposta de freqüência do sistema esteja relacionada linearmente com a perturbação, segundo uma equação diferencial linear $[9,11]$.

Se as condições iniciais do sistema forem todas iguais a zero, define-se a impedância eletroquímica $Z$, como sendo o quociente:

$$
Z(s)=\frac{E(s)}{I(s)}=\frac{s^{n}+a_{s-1} s^{n-1}+\ldots+a_{1} s+a_{0}}{b_{m} s^{n}+b_{m-1} s^{m-1}+\ldots+b_{1} s+b_{0}},
$$

onde $E(s)$ e $I(s)$ são, respectivamente, as transformadas de Laplace do potencial e da corrente e $s=j w, j^{2}=-1$, é a freqüência complexa de Laplace $[7,11]$.

\footnotetext{
${ }^{1}$ Departamento de Físico-Química - Instituto de Química - Unesp - CEP 14801-970 - Araraquara - SP -Brasil
} 
A impedância eletroquímica $Z(s)$ é uma função da variável complexa e pode ser representada por:

$Z(j w)=Z_{1}(j w)+j Z_{2}(j w)$,

onde $Z_{1}(j w)=\operatorname{Re}[Z(j w)]$ e $Z_{2}(j w)=\operatorname{Im}[Z(j w)]$ são, respectivamente, as partes real e imaginária. Além dessa representação, tem-se também que a impedância pode ser dada pela magnitude /Z(jw)/ e pelo ângulo de fase $\phi(w)$ :

$Z(j w)=|Z(j w)| e^{j \phi(w)}$.

Em geral, a função de um modelo de impedância adequado para processos de eletrodo simples é deduzida de um modelo elétrico equivalente, denominado circuito de Randles [12]. Este trabalho tem por objetivo estudar a generalização dessa função, com o intuito de ajustála às medidas experimentais de impedância, obtidas de um sistema constituído por uma liga $\mathrm{T}_{\mathrm{i}}-10 \% \mathrm{Al}$ $(\mathrm{m} / \mathrm{m})$ em solução de cloreto de sódio $0,9 \%$, variando-se a amplitude de perturbação.

Dos materiais utilizados em implantes para aplicações ortopédicas e dentárias, as ligas à base de titânio são as mais apropriadas, porque têm apresentado melhores propriedades mecânicas, resistência à corrosão e boa biocompatibilidade $[5,10]$.

\section{Material e métodos}

\section{Preparação da Amostra / Medidas de Impedância}

A liga foi preparada utilizando-se alumínio e pastilhas de 22 gramas de titânio de grau 1 de alta pureza, fornecidas pela empresa alemã Dentaurum. A fundição foi realizada em atmosfera de argônio empregando-se o processo plasma-skull e uma máquina Discovery Plasma, EDG Equipamentos.

Após a fundição, os corpos de prova de Ti-10Al foram submetidos ao polimento mecânico com lixas d'água de granulação 600,1000 e 1500 mesh, constituindo-se assim os eletrodos de trabalho.

Uma célula eletroquímica convencional foi preparada com três eletrodos, sendo o contraeletrodo um cilindro de grafite de alta pureza e, como sistema de referência foi adotado o eletrodo de calomelano saturado.

Para as medidas de impedância eletroquímica foi utilizada uma Interface Eletroquímica 1287 Solartron, acoplada a um Analisador de Resposta de Freqüência 1255 Solartron, com amplitudes de perturbação no potencial de $1 \mathrm{mV}$ e $10 \mathrm{mV}$ r.m.s. e 5 pontos por década de freqüência no intervalo de $100 \mathrm{kHz}$ a 0,1 $\mathrm{Hz}$.

\section{Impedância eletroquímica para um processo de eletrodo simples}

A função que descreve o espectro de impedância de sistemas simples de corrosão, controlados somente por transferência de carga e os casos de corrosão uniforme sobre superfícies homogêneas, pode ser deduzida de um circuito elétrico equivalente e é dada por:

$Z\left(w, R_{\Omega}, R_{P}, C\right)=R_{\Omega}+\frac{R_{P}}{1-j C R_{P} w}$,

onde $R_{\Omega}$ representa todas as resistências ôhmicas, C a capacitância da superfície do eletrodo e a resistência de polarização [12].

Substituindo a função (5) na equação (2), expandindo em frações parciais e aplicando a transformada inversa de Laplace, obtém-se a resposta da corrente $\mathrm{i}(\mathrm{t})$ dada no domínio do tempo por [9]:

$$
\left|i(t)=e^{\frac{R_{\alpha}-R_{p}}{R_{p} R_{p} C^{\prime}}}+\delta E\right| \frac{1}{F\left(w, R_{\Omega}, R_{p}, C\right)} \mid \operatorname{sen}\left(w t+\operatorname{arctg}\left(R_{p} C w\right)\right)
$$

Se o sistema for estável, então a resposta (6) deverá ser limitada. Isto significa que o termo exponencial deverá ser decrescente, ou seja, deverá ser válida a seguinte condição:

$$
\frac{R_{\Omega}+R_{P}}{R_{\Omega} R_{P} C}<0
$$

A equação (6) é a resposta de estado estacionário do sistema eletroquímico ao sinal senoidal de entrada (1), conhecida como resposta de freqüência do sistema. Se o sistema for estável, o sinal de saída difere da forma de onda do sinal de entrada somente no que diz respeito à amplitude e ao ângulo de fase. 


\section{Modelo de regressão}

Supondo que tenham sido realizadas $\mathrm{N}$ medições experimentais de impedância e que a função do modelo seja dependente de um vetor $\theta$ de $\mathrm{P}$ parâmetros, tem-se que o m-ésimo componente da impedância, $\mathrm{Z}_{\mathrm{m}}\left(\mathrm{w}_{\mathrm{K}}, \boldsymbol{\theta}\right), \mathrm{k}=1,2, \ldots, \mathrm{N} ; \mathrm{m}=1,2$, é uma variável aleatória dada por:

$Z\left(w_{k}, \theta\right)=Z_{1}\left(w_{k}, \theta\right)+j Z_{2}\left(w_{k}, \theta\right)$,

onde $\mathrm{j}^{2}=-1$ e $\mathrm{Z}_{1}\left(\mathrm{w}_{\mathrm{k}}, \theta\right)$ e $\mathrm{Z}_{2}\left(\mathrm{w}_{\mathrm{k}}, \theta\right)$ possuem um termo determinístico, dado pela função do modelo e uma variável aleatória, denominada erro residual.

Assim, escrevendo a parte determinística e os erros residuais, tem-se que o modelo (8) é equivalente a:

$Z\left(w_{1}, \theta\right)=\left(f_{1}\left(w_{k}, \theta\right)+j f_{2}\left(w_{k}, \theta\right)\right)+\left(\varepsilon_{1}\left(w_{k}, \theta\right)+j \varepsilon_{2}\left(w_{k}, \theta\right)\right), k=1, \ldots, N .(9)$

As funções $f_{m}, m=1,2$ e $\varepsilon_{m}, m=1,2$ são, respectivamente, as partes real e imaginária da função do modelo e do termo do erro aleatório.

Os erros residuais do modelo de regressão (9) possuem uma contribuição determinística (sistemática) e uma contribuição estocástica, distribuída aleatoriamente. Os erros estocásticos são inevitáveis em qualquer conjunto de dados experimentais. Os erros sistemáticos são compostos por erros originários de um modelo inadequado (falte de ajuste) e por erros tendenciosos, devido às limitações dos equipamentos e ao comportamento não estacionário do sistema eletroquímico. Geralmente a contribuição dos erros de instrumento é observada em freqüências altas e a contribuição não-estacionária, em freqüências baixas, que exigem muito tempo para as medições $[1,2,3]$.

Considerando-se somente a contribuição estocástica, o erro é uma variável aleatória complexa. Os estimadores dos parâmetros serão dados pelos valores $\theta$ de que otimizam algum critério sobre os erros residuais $\varepsilon(w, \theta)$.

Supondo os termos do erro com distribuição normal de média 0 , correlacionados em uma mesma freqüência e independentes para freqüências diferentes, isto é:

Ecl. Quím., São Paulo, 28(1): 63-67, 2003
$E\left[\varepsilon_{m}\left(w_{k}, \theta\right)\right]=0 \quad \mathrm{e}$

$E\left[\varepsilon_{m}\left(w_{k}, \theta\right) \varepsilon_{i}\left(w_{r}, \theta\right)\right]=\left\{\begin{array}{lll}\sum l_{m} & \text { se } & k=r \\ 0 & \text { se } & k \neq r\end{array}\right.$

onde E é o valor esperado e $\Sigma$ é a matriz $2 \times 2$ de covariâncias, o critério de estimação de parâmetros consiste em se determinar o vetor de parâmetros que minimiza o determinante da matriz $\varepsilon^{t} \varepsilon$, o que corresponde a minimizar a função [6]:

$D(\theta)=\sum_{k=1}^{N} \varepsilon_{1}^{2}\left(w_{k}, \theta\right) \sum_{k=1}^{N} \varepsilon_{2}^{2}\left(w_{k}, \theta\right)-\left(\sum_{k=1}^{N} \varepsilon_{1}\left(w_{k}, \theta\right) \varepsilon_{2}\left(w_{k}, \theta\right)\right)^{2}$.

Os intervalos de confiança para o parâmetro $\theta_{r}$ são calculados por

$\hat{\theta_{r}} \pm t(N-p ; \beta / 2) s \sqrt{2\left\{\Omega^{-1}\right\}_{r r}}$

onde $\hat{\theta}_{r}$ é a estimativa do parâmetro, $\mathrm{t}(\mathrm{N}-\mathrm{p}, \beta / 2)$ é o valor da distribuição de Student ao nível $\beta$ de significância com $\mathrm{N}$ - p graus de liberdade, $s^{2}=D(\hat{\theta}) / N-p$ e $\Omega$ é a matriz hessiana [6].

\section{Resultados e discussão}

Os resultados experimentais de impedância de uma interface eletroquímica, na maioria das vezes, não são explicados por um modelo linear consistindo somente dos parâmetros de um circuito elétrico equivalente.

Com o objetivo de minimizar essa distorção entre a curva experimental e a curva teórica, considerou-se a generalização da função (5), definida por:

$$
Z_{\alpha}\left(w, R_{\Omega}, R_{p}, C, \alpha\right)=R_{\Omega}+\frac{R_{p}}{\left(1-j R_{p} C w\right)^{\alpha}}
$$

Se o parâmetro $\alpha$ for igual a 1 , então a função (13) resulta na função (5), $Z\left(w, R_{\Omega}, R_{P}, C\right)$, e os resultados são consistentes com a teoria de sistemas lineares.

Escrevendo a forma polar da função (13) verifica-se que o parâmetro $\alpha$ está relacionado com o módulo e com o ângulo de fase da impedância:

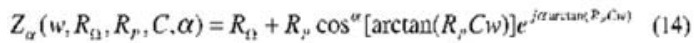

O parâmetro $\alpha$ é equivalente ao elemento de fase fracional definido por de Levie [8] e possui a mesma interpretação física do elemento de fase constante. Para $\alpha=0,5$ o eletrodo é poroso e para $\alpha=1 \quad$ o eletrodo é plano e polido. Valores intermediários de, $\alpha, 0,5<\alpha<1$, correspondem a interfaces 
rugosas $[7,8,12]$.

A função $Z_{\alpha}\left(w, R_{\Omega}, R_{P}, C, \alpha\right)$ foi ajustada às medidas experimentais de impedância obtidas de um sistema constituído de uma liga de Titânio-Alumínio (Ti-10Al) em solução de cloreto de sódio $0,9 \%$ no potencial de $1 \mathrm{~V}$, com aplicação de $1 \mathrm{mV}$ e $10 \mathrm{mV}$ de amplitude de perturbação, na faixa de freqüência de $100 \mathrm{kHz}$ a $0,1 \mathrm{~Hz}$.

$\mathrm{Na}$ tabela 1 são apresentados os resultados e os intervalos de confiança das estimativas dos parâmetros função $Z_{\alpha}\left(w, R_{\Omega}, R_{P}, C, \alpha\right)$.Como a estimativa do parâmetro $R_{\Omega}$ apresentou excessivo erro relativo, este foi fixado em 60, observando-se o comportamento limite da parte real em altas freqüências.

\begin{tabular}{|c|c|c|c|c|}
\hline & $R_{\Omega}\left(\Omega \mathrm{cm}^{-2}\right)$ & $R_{p}\left(\Omega \mathrm{cm}^{-2}\right)$ & $R_{p} C\left(f \mathrm{~cm}^{-2}\right)$ & $\alpha$ \\
\hline $\begin{array}{c}\text { Amplitude de } \\
1 \mathrm{mV} \\
\end{array}$ & $\begin{array}{c}60 \\
\text { (Fixo) } \\
\end{array}$ & $\begin{array}{c}4,017 \times 10^{5} \\
\pm \\
1.88 \times 10^{4}\end{array}$ & $\begin{array}{c}3,155 \\
\pm \\
0,22\end{array}$ & $\begin{array}{c}0,955 \\
\pm \\
0,018\end{array}$ \\
\hline $\begin{array}{l}\text { Amplitude de } \\
10 \mathrm{mV}\end{array}$ & $\begin{array}{c}60 \\
\text { (Fixo) }\end{array}$ & $\begin{array}{c}3,236 \times 10^{5} \\
\pm \\
1,35 \times 10^{3}\end{array}$ & $\begin{array}{c}3.545 \\
\pm \\
0.028\end{array}$ & $\begin{array}{l}0,92 \\
\pm \\
1,71 \times 10^{-7}\end{array}$ \\
\hline
\end{tabular}

Tabela 1: Resultados das estimativas dos parâmetros de $\mathrm{F}_{\alpha}\left(\mathrm{W}, R_{\Omega}, \mathrm{R}_{\mathrm{P}}, \mathrm{C}, \alpha\right)$ e respectivos intervalos de confiança para as amplitudes de $1 \mathrm{mV}$ e $10 \mathrm{mV}$.

Os resultados observados na tabela 1 mostram que as estimativas obtidas para a amplitude de perturbação de $10 \mathrm{mV}$ apresentam intervalos de confiança menores que aqueles obtidos com a amplitude de $1 \mathrm{mV}$. O parâmetro apresentou estimativa mais próxima de 1 para o caso de $1 \mathrm{mV}$, como era esperado para sistemas sujeitos a perturbações pequenas. Contudo, a condição (7) não é verificada, ou seja, o sistema é instável para essa amplitude de perturbação. Essa instabilidade pode ter influenciado a falta de ajuste do modelo.

As figuras 1 e 2 ilustram respectivamente a representação no plano complexo das medidas experimentais para a amplitude de $1 \mathrm{mV}$ e de $10 \mathrm{mV}$ e seus correspondentes valores teóricos.

$\mathrm{Na}$ figura 3 estão representados os erros residuais, determinados pela diferença entre os valores experimentais e os valores teóricos.

Comparando-se os gráficos das figuras 1 e 2 e observando-se o gráfico dos erros residuais da figura 3 , verifica-se que a qualidade do ajuste é melhor para o espectro de impedância obtido com a perturbação de $10 \mathrm{mV}$.

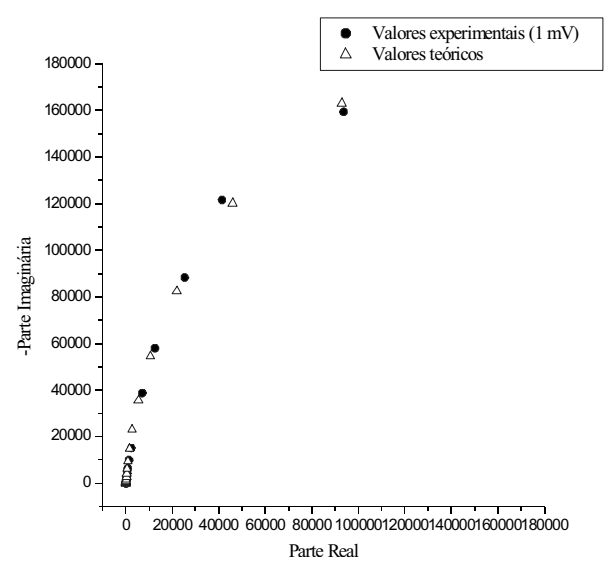

Figura 1. Representação no plano complexo das medidas experimentais e dos valores teóricos calculados com a função $Z_{\alpha}\left(w, R_{\Omega}, R_{P}, C, \alpha\right)$ para a amplitude de $1 \mathrm{mV}$.

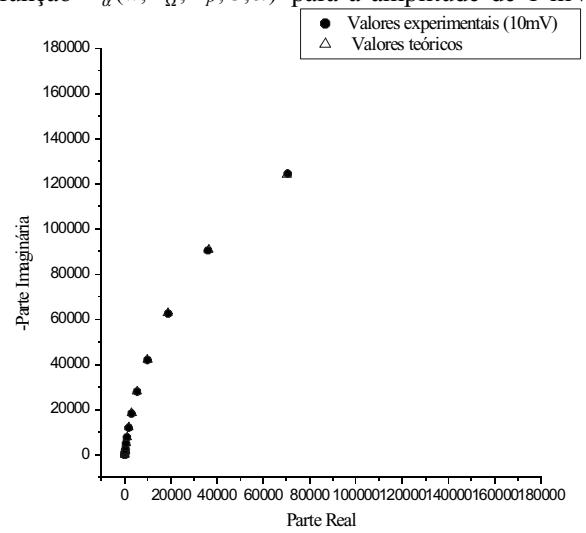

Figura2. Representação no plano complexo das medidas experimentais e dos valores teóricos calculados com a função $Z_{\alpha}\left(w, R_{\Omega}, R_{P}, C, \alpha\right)$ para a amplitude de $10 \mathrm{mV}$.

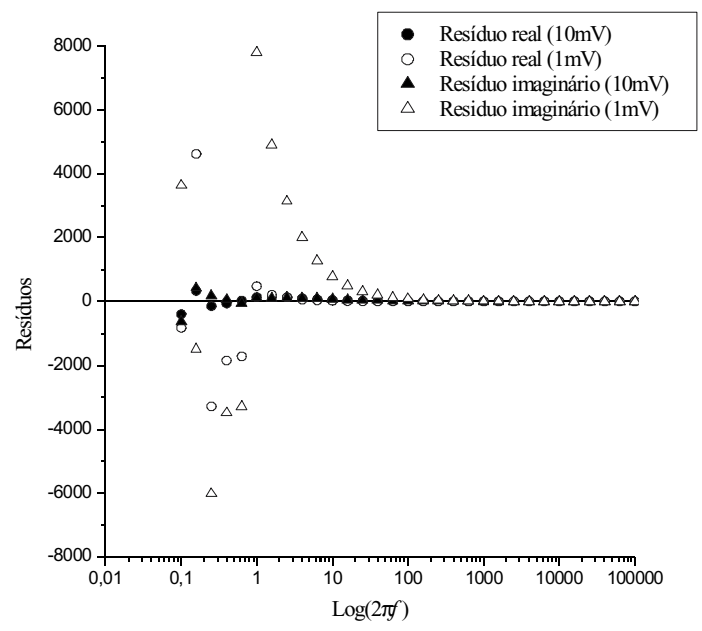

Figura 3. Representação gráfica dos erros residuais para as amplitudes de $1 \mathrm{mV}$ e $10 \mathrm{mV}$.

Ecl. Quím., São Paulo, 28(1): 63-67, 2003 


\section{Conclusões}

A análise dos resultados indica que a função $Z_{\alpha}$ é adequada para descrever a impedância do sistema analisado. O parâmetro $\alpha$ confere flexibilidade ao ângulo de fase e ao módulo da impedância, contribuindo assim para a redução das distorções entre a curva experimental e a curva teórica.

Além disso, os melhores resultados de ajuste foram obtidos para sinais de perturbação do sistema com amplitude igual a $10 \mathrm{mV}$. Diminuindose a amplitude para $1 \mathrm{mV}$ o comportamento do sistema aproximou-se mais do comportamento linear, contudo os resultados foram influenciados pela instabilidade do sistema.

Portanto, a análise de resposta de freqüência do sistema deve ser realizada para uma amplitude de perturbação na qual o comportamento do sistema não se afaste muito do linear, porém o suficiente para não serem observados os efeitos da instabilidade.

CAPELA, J. M. V. et al. Generalization of a linear model of electrochemical impedance.

\section{Abstract}

The function of impedance for processes of simple electrode is generally deduced from the Randles equivalent electric circuit. In this work the generalization of this function was studied, by the introduction of a parameter related to the flexibility of its phase angle and magnitude. The function was fitted to the impedance experimental measures from the Ti-10wt.\%Al alloy in sodium chloride solution $0.9 \%$ and varying the amplitude of perturbation. The results showed that the generalized function reduces the distortions between the experimental and the theoretical curve with the best results obtained for signals with amplitude of $10 \mathrm{mV}$.

Keywords: Electrochemical impedance; Linear systems; Non linear regression; Titanium alloys.

\section{Referências bibliográficas}

[1] AGARWAL, P.; CRISAlle, O. D.; ORAZEM, M. E. Application of measurement models to impedance spectroscopy: II Determination of the stochastic contribution to the error structure. J. Electrochem. Soc., v. 142, n. 12, p. 4149-4158, 1995.

[2] AGARWAL, P.; MOGHISSI, O. C; ORAZEM, M. E.; GARCIA-RUBIO, L. H. Application of measurement models for analysis of impedance spectroscopy. Corrosion, v. 49 , p. 278-289, 1993.

[3] AGARWAL, P.; ORAZEM, M. E. Application of measurement models to impedance spectroscopy: III Evaluation of consistency with the Kramers-Kronig relations. $J$. Electrochem. Soc., v. 142, n. 12, p. 4159-4168, 1995.

[4] ARMSTRONG, R. D.; FIRMAN, R. E.; THIRSK, H. R. The A. C. impedance of complex electrochemical reactions. Disc. Farad. Soc., v. 56, p. 244-263, 1973.

[5] AZIZ-ERRZO, M.; CONRAY, K. G.; FENELON, A. M.; FARELL, S. T.; BRESLIN,C. B. Electrochemical studies on the stability and corrosion resistance of titaniumbased implant materials. Biomaterials, v. 22, p. 1531-1539, 2001

[6] BATES, D. M.; WATTS, D. G. Nonlinear regression analysis and its applications. New York: John Wiley, 1988. $365 \mathrm{p}$.

[7] BRETT, A. M. O.; BRETT, C. M. A. Electroquímica, princípios, métodos e aplicações. Coimbra: Almedina, 1996. $471 \mathrm{p}$.

[8] DE LEVIE, R. On impedance of electrodes with rough interfaces. J. Electroanal. Chem., v. 261, p.1-9, 1989

[9] DORF, R. C.; BISHOP, R. H. Sistemas de controle modernos, Rio de Janeiro: LTC, 2001. 659p.

[10] LAYANI,J. D.; MAYER, L.; CUISINIER, F. J. G. Carbonated hydroxyapatites precipitated in the presence of Ti. J. Inorg. Biochem., v. 81, p. 57-63, 2000.

[11] MACDONALD, D. D.; SIKORA, E.; ENGELHARDT, G. Characterizing electrochemical systems in the frequency domain. Electrochim. Acta, v. 43, p. 87-107, 1998.

[12] MANSFELD, F.; SHIH, H.; GREENE, H.; TSAI, C. H. Analysis of EIS data for common corrosion processes. In: SCULLY, J. R.; SILVERMAN, D. C.; KENDIG, M. W. Electrochemical impedance: analysis and interpretation. Philadelphia: ASTM, 1993. p. 37-53.

Recebido em: 18/12/02

Aceito em: 23/04/03 